\title{
A new top jet tagging algorithm for highly boosted top jets
}

\section{Salvatore RAPPOCCIO*}

Department of Physics andAstronomy, Johns Hopkins University, Baltimore, MD, 21218, USA

E-mail: rappoccio@gmail.com

\section{For The CMS Collaboration}

\begin{abstract}
A new top-jet-tagging algorithm is presented based on work in Ref [1]. The full results can be seen in Refs $[2,3]$. This algorithm uses the Cambridge-Aachen jet clustering algorithm to decompose highly boosted top jets into subjet components and examine the kinematics of these subjets. With this algorithm, an efficiency of $46 \%$ for top-jets with $p_{\mathrm{T}}=600 \mathrm{GeV} / c$ is obtained, together with a rejection of $98.5 \%$ for non-top jets with $p_{\mathrm{T}}=600 \mathrm{GeV} / c$.

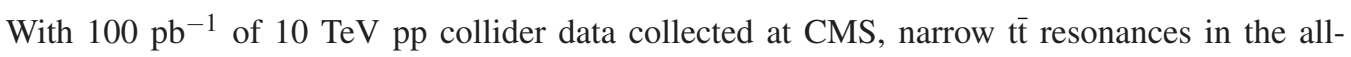
hadronic channel, with masses of 1, 2, 3, and $4 \mathrm{TeV} / \mathrm{c}^{2}$ can be excluded at the $95 \%$ C.L. for cross sections of $17.2,1.5,0.7$, and $0.8 \mathrm{pb}$, respectively. A $5 \sigma$ discovery can be made for cross sections of $43.6,4.0,1.6$, and $1.3 \mathrm{pb}$, respectively.
\end{abstract}

European Physical Society Europhysics Conference on High Energy Physics

July 16-22, 2009

Krakow, Poland

${ }^{*}$ Speaker. 
Top quarks play an important role in electroweak symmetry breaking scenarios because of the large coupling to the Higgs field compared to other quarks. Moreover, many theoretical extensions of the Standard Model contain new particles that decay into top quarks with a large branching fraction, including Randall-Sundrum KK gluons. If these new particles are sufficiently massive, the resulting top quarks are highly boosted, and may collapse into a single jet. It is therefore useful to develop reconstruction algorithms that attempt to distinguish these boosted top quarks from those produced in the generic QCD background from proton-proton collisions.

The general strategy for tagging boosted top quarks decaying hadronically is to identify jet substructure in top-quark jets, and to use this substructure to impose kinematic cuts that discriminate against non-top jets of the same transverse momentum. To accomplish this, the CambridgeAachen [4] jet clustering algorithm is used on the electromagnetic calorimeter (ECAL) and the hadron calorimeter (HCAL) of CMS [5], in towers of size $(0.087,0.087)$ in the (rapidity-azimuth) coordinates in the central barrel region. The hierarchical clustering sequence of the construction of the jets is used to extract "subjets" that originate from the quarks in the top quark's hadronic decay chain.

The masses involved in the top decay chain (the top mass and the $\mathrm{W}$ mass) provide powerful discrimination against generic non-top jets. The efficiency obtained for this algorithm is shown in Figure 1a. The top tagging algorithm achieves an efficiency of $46 \%$ for top-jets with $p_{\mathrm{T}}=600$ $\mathrm{GeV} / c$, together with a rejection of $98.5 \%$ for non-top jets with $p_{\mathrm{T}}=600 \mathrm{GeV} / c$.

Using generic dijets as a signal-depleted sample, a data-driven background parameterization can be derived. This parameterization can be used to perform searches for new tit resonances in the all-hadronic channel. The total background for a general di-jet search in the $t \bar{t}$ all-hadronic channel is shown in Figure 1b.

With $100 \mathrm{pb}^{-1}$ of $10 \mathrm{TeV}$ pp collision data collected at CMS, narrow tt resonances in the allhadronic channel, with masses of $1,2,3$, and $4 \mathrm{TeV} / c^{2}$ can be excluded at the $95 \%$ C.L. for cross sections of $17.2,1.5,0.7$, and $0.8 \mathrm{pb}$, respectively. A $5 \sigma$ discovery can be made for cross sections of $43.6,4.0,1.6$, and $1.3 \mathrm{pb}$, respectively.

\section{References}

[1] David Kaplan, Keith Rehermann, Matthew Schwartz, and Brock Tweedie. Top-tagging: A Method for Identifying Boosted Hadronic Tops. Phys.Rev.Lett, 101:142001, 2008.

[2] A Cambridge-Aachen (C-A) based Jet Algorithm for boosted top-jet tagging. CMS PAS JME-009-01.

[3] Search for High-Mass Resonances Decaying into Top-Antitop Pairs in the All-Hadronic Mode . CMS PAS EXO-009-02.

[4] Y.L. Dokshitzer, G.D. Leder, S. Moretti, and B.R. Webber. Better Jet Clustering Algorithms. JHEP, 9708:001, 1997.

[5] S Chatrchyan et al. The CMS experiment at the CERN LHC. Journal of Instrumentation, 3(08):S08004, 2008. 


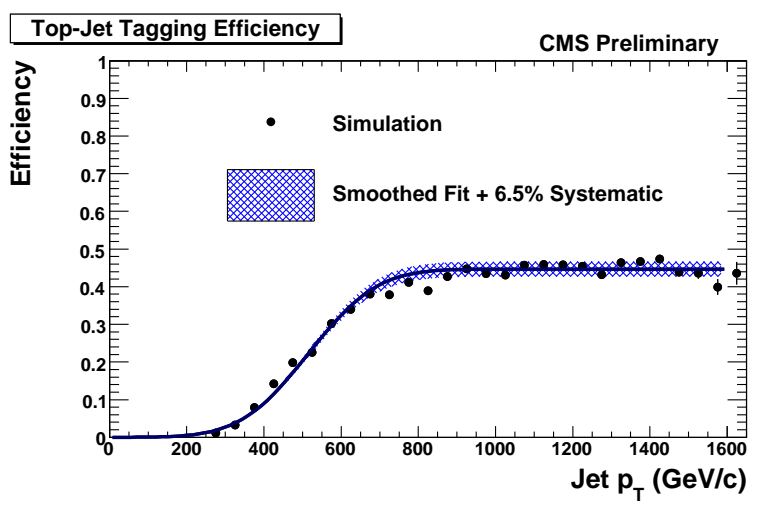

(a) Efficiency for matched top-jets, including the $6.5 \%$ systematic uncertainty.

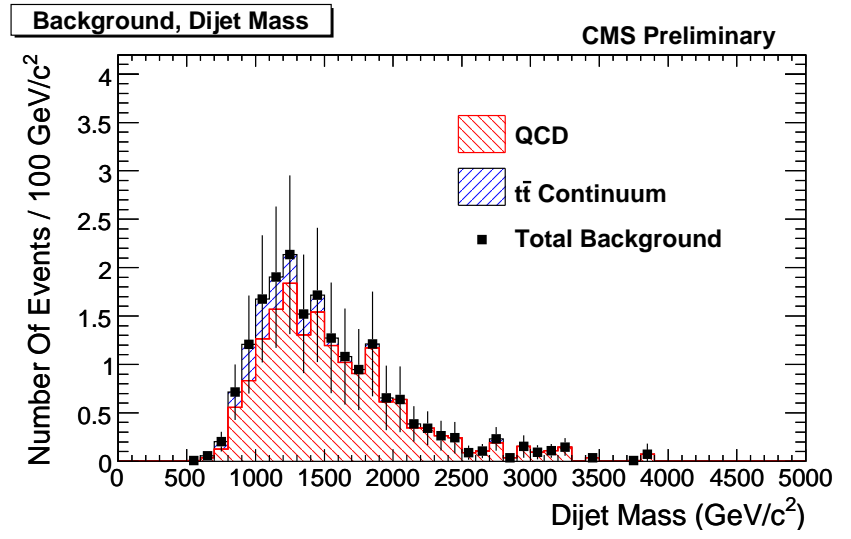

(b) Total background estimate for the double-tag analysis. The continuum $\mathrm{t} \overline{\mathrm{t}}$ contribution is shown in $45^{\circ}$ hatching, and the QCD dijet contribution is shown in $-45^{\circ}$ hatching.

Figure 1: Performance of top tagging. 